\title{
Prävention mit Vitaminen und Spurenelementen
}

Prof. Dr. A.F.H. Pfeiffer ${ }^{1,2}$, Dr. Ch. Einig ${ }^{1}$

${ }^{1}$ Deutsches Institut für Ernährungsforschung, Bergholz-Rehbrücke

${ }^{2}$ Abt. f. Endokrinol., Diabetes u. Ernährungsmed., Universitätsklinikum Benjamin Franklin, Berlin

\section{Disease prevention by vitamins and trace elements}

Vitamins and trace elements are largely provided by a balanced nutrition. In industrialized countries, though, frequent deficencies occur e.g. in folic acid and vitamin $D$ as well as iodide, iron and calcium. A short review of recommended daily intake is presented.

Der Bedarf an Vitaminen und Spurenelementen kann weitgehend durch gesunde Ernährung gedeckt werden. Einzelne Nahrungskomponenten werden jedoch häufig nicht ausreichend durch Nahrung zugeführt oder sollten in bestimmten Situationen, wie z. B. Schwangerschaft, supplementiert werden. Die Substitution von Folsäure und Vitamin D ist in der Literatur gesichert; zu anderen Vitaminen liegen keine ausreichenden Daten vor. Grundsätzlich wird die „five a day"-Regel, also 5 Portionen Gemüse/Obst am Tag, empfohlen.

Drei große Studien, zuletzt die Heart Protection Study mit 20536 Teilnehmern (Lancet 2002, 360: 23-33) in England, konnten auch bei Risikopatienten keinen Nutzen durch die Einnahme der antioxidativen Vitamine A, C und E nachweisen, so dass für deren Supplementation kaum Argumente vorliegen, während Querschnittstudien eine höhere Krankheitsinzidenz mit niedrigen Vitamin-C-Spiegeln zeigen (Lancet 2001, 357: 657 - 663).

\section{Folsäure}

Die Daten des Bundesgesundheitssurvey 1998 zeigen, dass die Folsäureversorgung bei $80 \%$ der untersuchten Frauen zwischen 18 und 40 Jahren suboptimal ist. Für das Auftreten von Neuralrohrdefekten ist eine ungenügende Folsäureversorgung in der Frühschwangerschaft mitverantwortlich. Neuralrohrdefekte treten bei 0,1-0,2 $\%$ aller Schwangerschaften und Geburten auf. Der Folsäurebedarf wird generell und besonders in der Schwangerschaft mit den in Deutschland üblichen Ernährungsgewohnheiten kaum gedeckt. Seit 1994 empfehlen Fachgesellschaften deshalb
Frauen, die nicht gezielt verhüten oder ein Kind wünschen, die Einnahme von $0,4 \mathrm{mg} / \mathrm{d}$ Folsäure zusätzlich zur Nahrung. Für Frauen, die schon durch eine Schwangerschaft mit Neuralrohrdefekt vorbelastet sind, hat sich eine Dosis von $4 \mathrm{mg} / \mathrm{d}$ als effektiv erwiesen. Die Therapie sollte mindestens einen Monat vor der Konzeption beginnen und wenigstens die ersten 3 Schwangerschaftsmonate andauern. Eine Supplementierung innerhalb der ersten 4 Wochen der Schwangerschaft scheint ebenfalls noch präventiv zu sein. Die Missbildungstendenz kann durch perikonzeptionelle Folsäuregaben in einer Dosierung von $0,4-4 \mathrm{mg} / \mathrm{d}$ um $50-80 \%$ reduziert werden (Hages, M. et al. Geburtsh Frauenheilk 1996; 56: M59 - M65). Folsäure ist neben den Vitaminen $B_{6}$ und $B_{12}$ beim $A b-$ bau von Homocystein beteiligt. Störungen bei der Homocysteinmetabolisierung werden nicht nur u.a. für Neuralrohrdefekte diskutiert, sondern auch als ein potenzieller Risikofaktor für die Entstehung der Arteriosklerose. Zur Senkung erhöhter Homozysteinspiegel wird zur Zeit eine regelmäßige tägliche Folataufnahme mit der Nahrung in Höhe von 0,4 mg empfohlen. Gute Folatquellen sind u.a. Tomaten, Gurken, Kohl, Kartoffeln, Orangen und Weizenkeime.

\section{Eisen}

Ursache für einen schweren Eisenmangel sind zumeist chronische Blutverluste. Eisenmangel führt u.a. zur hypochromen Anämie, reduzierter körperlicher Leistungsfähigkeit, erhöhter Infektanfälligkeit und gestörter Thermoregulation. In der Schwangerschaft sowie auch während des Wachstums ist der Eisenbedarf erhöht.
Risikogruppen für Eisenmangel sind u.a. Säuglinge, Kinder und Jugendliche im Wachstum, stark menstruierende Frauen, Schwangere, Senioren und Veganer. Wegen der geringen Verfügbarkeit von Eisen aus der Nahrung empfiehlt die Deutsche Gesellschaft für Ernährung eine Zufuhr von 15 mg/d für Frauen vor der Menopause und $10 \mathrm{mg} / \mathrm{d}$ für die anderen Erwachsenen. Wegen des erhöhten Bedarfs in der Schwangerschaft sollte die Zufuhr bei $30 \mathrm{mg} / \mathrm{d}$ liegen, was sich aber in der Regel nicht durch die Nahrung verwirklichen lässt. Im Gegensatz zur Bioverfügbarkeit von Häm-Eisen aus tierischen Lebensmitteln (ca. 20\%) liegt die des Nicht-Häm-Eisens aus pflanzlichen Lebensmitteln (Gemüse, Getreideprodukte) nur bei ca. $5 \%$. Sie wird z.B. durch Oxalate (in Rharbarber, Spinat), Phytinsäure (in Getreide, Hülsenfrüchten), Tannine (schwarzer Tee, Kaffee) und bestimmte Medikamente vermindert. Vitamin C und Zitronensäure (Früchte) sowie Milchsäure wirken resorptionsfördernd. Im Mangel steigt die Absorptionsrate auf das 2-3fache.

Eine zu hohe Eisenzufuhr wird heutzutage im Zusammenhang mit radikal-assoziierten Erkrankungen (Koronar- und Tumorerkrankungen) diskutiert. Bei längerfristigen Gaben ab 100 mg Eisen/d (LOAEL - Lowest Observed Adverse Effect Level) können Nebenwirkungen auftreten (Hathcock, JN. Vitamin and mineral safety. Council for Responsible Nutrition, Washington DC 1997).

Jod

Das Ziel, den Jodmangel bis zum Jahr 2000 zu beseitigen, hat Deutschland im Gegensatz zu anderen Ländern nicht erreicht. Jährlich werden als Folge des Jod- 
rung teilweise die Symptomatik verstärken (Hahn, A. Nahrungsergänzungsmittel Wiss. Verl.-Ges. Stuttgart 2000:174-176).

\section{Calcium und Vitamin D}

Die Calcium-Zufuhr bestimmt, im genetisch vorgegebenen Rahmen, während des pubertären Wachstumsschubes die „peak bone mass" sowie, im fortgeschrittenen Alter, die Geschwindigkeit des Knochenmasseverlustes durch Hemmung der Parathormonsekretion. Empfehlungen beruhen auf Calcium-Bilanzrechnungen und epidemiologischen, teilweise prospektiven Studien der Knochenkalkmasse und Frakturhäufigkeiten. Langfristige Untersuchungen der Calcium-Aufnahme als Kind für die Knochenmasse und späteres Osteoporoserisiko fehlen. Empfohlen werden 0,8-1 g Calcium/d für 6-10-Jährige, danach 1,3-1,5 $\mathrm{g} / \mathrm{d}$ bis zum 18. Lebensjahr, worunter akzeptable Calcium-Bilanzen auftraten (Martin et al., 1997 Am J Clin Nutr 66: 611).

Bei postmenopausalen Frauen wurde eine Reduktion der Frakturrate und eine Verbesserung der Knochendichte durch Calcium-Supplemente bei Frauen mit niedriger Calciumzufuhr in kontrollierten Studien gezeigt, während große populationsbasierte Studien keine Abnahme der Frakturrate mit steigender Calciumzufuhr nachweisen konnten (Cummings et al., 1997 Am J Epidemiol 145: 926). Für Männer liegen vergleichbare Daten vor. Eine Calciumzufuhr von 1200 $\mathrm{mg} / \mathrm{d}$ ist die aktuelle empfohlene Dosis. Sie hemmt die im Alter gesteigerte Parathormonsekretion und erzielt bei Gesunden eine ausgeglichene Calciumbilanz ohne zusätzliches Vitamin D.

In Deutschland ist jedoch aufgrund eines lange dauernden Jodmangels vielfach mit unerkannten funktionellen Autonomien der Schilddrüse, insbesondere bei Senioren, zu rechnen. Deshalb sollte die tägliche alimentäre Jodzufuhr bei Erwachsenen $500 \mathrm{mg} / \mathrm{d}$ nicht überschreiten (Dtsch Ges. für Ernährung et al. Referenzwerte für die Nährstoffzufuhr. Umschau/Braus, Frankfurt/Main 2000:179-183). Bei Hyperthyreose kann eine Jodsupplementie-
Phytat und Oxalat in der natürlichen Nahrung sowie Cola-Getränke reduzieren die Resorption. Calcium-Supplemente werden gleich gut wie natürliches Nahrungscalcium aufgenommen. Calcium-Zitrat hat eine geringfügig höhere Bioverfügbarkeit als Calcium-Carbonat; wichtig ist vor allem eine gute Löslichkeit. Calcium-Carbonat wird bei Achlorhydrie schlecht resorbiert und reduziert die Eisenaufnahme, weshalb es mit einer eisenarmen Mahlzeit, üblicherweise dem Frühstück, eingenommen werden sollte. Calcium-Nierensteine werden bei höherer Calciumzufuhr seltener beobachtet. Wahrscheinlich wird die Oxalatresorption durch Calciumkomplexbildung reduziert, besonders wenn Calcium mit der Nahrung zugeführt wird. Eine Obergrenze liegt bei $2,5 \mathrm{~g} / \mathrm{d}$ Calcium, darüber treten Nebenwirkungen wie Obstipation auf. Neben Milchprodukten sind calciumreiches Wasser, Brokkoli, Lauch, Fenchel und Grünkohl gute Calciumquellen.

Die Calcium-Resorption wird durch Parathormon und Vitamin D reguliert. Vitamin-D-Mangel besteht in unseren Breiten in den Wintermonaten. Menschen mit dunkler Hautfarbe und minimaler Sonnenexposition durch Bekleidungsgewohnheiten (z.B. Ältere, türkische und arabische Frauen) haben häufig Vitamin D-Mangel. Die Calciumresorption wird durch Vitamin D deutlich gesteigert. Die empfohlene tägliche Zufuhr für Gesunde beträgt $400 \mathrm{IU}$ und kann durch zwei Mahlzeiten Seefisch pro Woche leicht gedeckt werden.

Fazit: Neben einem endemischen Jodmangel findet sich häufig eine Defizienz von Folsäure, Vitamin D, Calcium und auch Eisen. Die Supplementierung bei ungünstigen Ernährungsgewohnheiten kann krankheitspräventiv sein.

Korrespondenz: Prof. Dr. A.F.H. Pfeiffer Deutsches Institut für Ernährungsforschung, A.-Scheunert-Allee 114-116, 14558 Bergholz-Rehbrücke

Fachliche Betreuung der
„Serie Prävention“:
Prof. Dr. Dr. h. c. Peter C. Scriba
Medizinische Klinik / Klinikum Innenstadt
der Universität München
Ziemssenstr. 1
80336 München
Prof. Dr. Friedrich W. Schwartz
Medizinische Hochschule Hannover
Abteilung für Epidemiologie, Sozialmedizin
und Gesundheitsforschung
Carl-Neuberg-Str. 1
30623 Hannover

